\title{
Striking nonlinear dynamics of mode-locked fibre lasers
}

\author{
Junsong Peng ${ }^{1}$, Sonia Boscolo ${ }^{2}$, Zihan Zhao ${ }^{1}$, and Heping Zeng ${ }^{1}$ \\ ${ }^{1}$ State Key Laboratory of Precision Spectroscopy, East China Normal University, Shanghai 200062, China \\ ${ }^{2}$ Aston Institute of Photonic Technologies, School of Engineering and Applied Science, Aston University, \\ Birmingham B4 7ET, United Kingdom \\ s.a.boscolo@aston.ac.uk
}

\begin{abstract}
We report on the real-time observation of various remarkable nonlinear phenomena in mode-locked fibre lasers. These include the build-up of dissipative solitons and soliton molecules, collision-induced soliton explosions, and the excitation and dynamics of breathing dissipative solitons and breather molecular complexes. Numerical simulations of the laser model support our experimental findings.
\end{abstract}

Keywords: dissipative nonlinear systems; mode-locked fibre lasers; pulse propagation and temporal solitons.

\section{INTRODUCTION}

During the last decades, the formation of localised wave structures has attracted significant research interest in a variety of conservative and dissipative systems of nonlinear science, including photonics, plasma physics, BoseEinstein condensates, fluid mechanics, biology, and chemistry. For instance, the notion of a soliton is one of the most well-known example of studies in which the initial concept has even been generalised to nonlinear dissipative systems, in which localised wave packets arise from the balance between dispersion or diffraction and nonlinearity (as the conventional solitons) and between gain and losses. Besides their formation, their mutual interactions such as collisions and even the emergence of stable bound states have been particularly investigated in laser physics [1]. Such dissipative multi-soliton complexes, also called soliton molecules, result from self-organisation phenomena and show dynamics similar to matter molecules, such as synthesis and vibration. The build-up and transient regimes of dissipative localised structures in mode-locked fibre lasers are currently the subject of intense studies by means of real-time ultrafast measurements [2-5]. Apart from parameter-invariant stationary solitons, numerous nonlinear systems support breathing dissipative solitons, manifesting themselves as nonlinear waves in which energy concentrates in a localised and oscillatory fashion. Similarly to Akhmediev and Kuznetsov-Ma breathers in conservative nonlinear systems, breathing dissipative solitons are related to the Fermi-Pasta-Ulam recurrence [6]. Besides their fundamental importance in nonlinear science, breathing solitons are also attractive because of their potential for applications, such as in spectroscopy.

In the present work, we review our recent results and advances in the area. By employing an ultrafast laser setup whose output is spectrally and temporally analysed in real time, we unveil various remarkable nonlinear dynamics of mode-locked fibre lasers. These include the multiple nonlinear processes involved in the build-up of dissipative solitons and soliton molecules [7,8], the dynamics of soliton molecules in the normal-dispersion regime of a laser cavity [9], a conceptually new type of soliton explosions arising from soliton collisions [10], the excitation and dynamics of breathing dissipative solitons and breather molecular complexes [11,12], and breather explosions [13]. Numerical simulations based on the complex Ginzburg-Landau equation or on an iterative cavity map confirm our experimental observations.

\section{DYNAMICS OF DISSIPATIVE SOLITONS}

\subsection{Build-up of solitons and soliton molecules}

The problem of the soliton formation mechanisms is of a fundamental physical interest. In contrast to nonlinear integrable models, the typical soliton formation dynamics in non-integrable systems are non-repetitive and exhibit complex behaviour before a stationary soliton settles down. Using the dispersive Fourier transform (DFT) technique and field autocorrelation analysis for the round-trip resolved measurements, we reveal the nontrivial laser dynamics leading to a stable mode-locking regime of a dissipative soliton fibre laser [7]. We find that the build-up of dissipative solitons depends critically on the nonlinearity accumulated inside the laser cavity. In a long cavity $(>10 \mathrm{~m})$ in which nonlinearity is high, several distinct physical processes are involved in the build-up phase (Fig. 1). During the initial stage of the evolution modulation instability gives rise to the formation of well-defined intensity patterns. On the next stage of the evolution, mode-locking starts and a soliton is formed. A subsequent stage of pulse splitting and pulse interactions is initiated by self-phase modulation instability. Finally, a stationary soliton emerges from a process of partial annihilation. Conversely, in a short cavity laser only modulation instability and mode locking are responsible for the formation of a stationary soliton.

In [8], we resolve the build-up of soliton-pair molecules in an ultrafast fibre laser. In particular, we reveal the formation of three types of molecules: ground- and excited-state soliton molecules (corresponding to close and wide intra-molecular temporal separations, respectively), and a conceptually new molecule type, which represents the intermediate state between a vibrational and a stationary molecule, hence we name 'intermittent- 
vibration soliton molecule'. We find that the first two stages of the build-up phase (mode locking and soliton splitting) are similar for the three molecules, but the final stage (soliton interactions) features different. For the ground-state molecule (Fig. 2, left panel) a variety of interactions take place, including attraction, repulsion, vibration and annihilation, whereas for the excited-state and intermittent-vibration molecules (Fig. 2, right panel) repulsive interactions dominate.
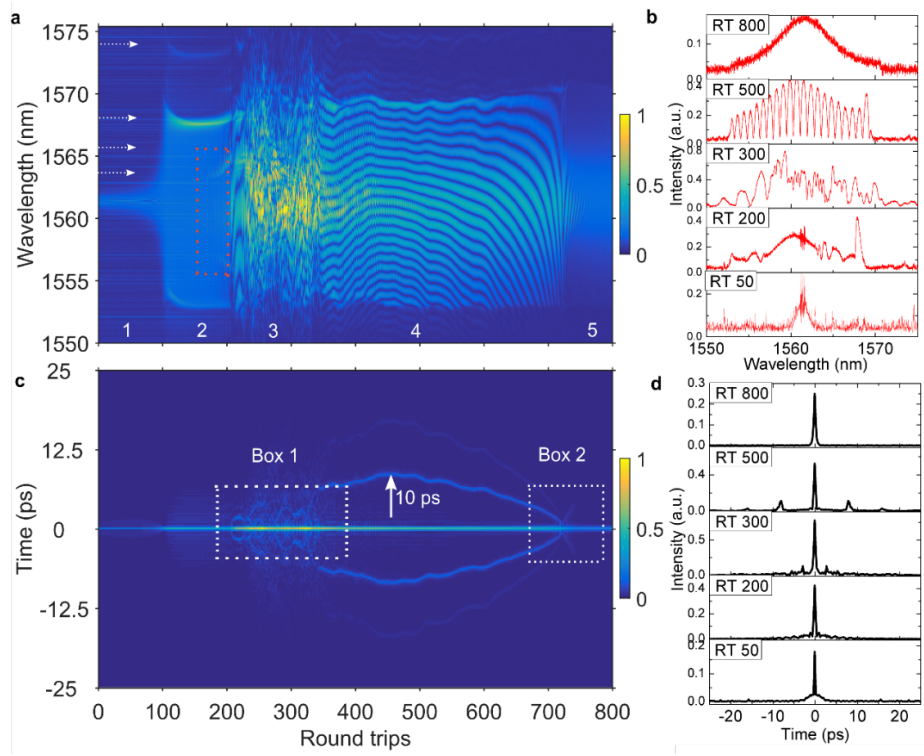

Figure 1. Build-up of dissipative solitons. (a) Real-time spectral evolution of the laser output during the build-up phase. (b) Typical cross-sections of (a). (c) The Fourier transforms of the single-shot spectra in (b) represent the field autocorrelations of the laser output, tracing the evolution of the temporal separations between solitons. (d) Representative cross-sections of (c).
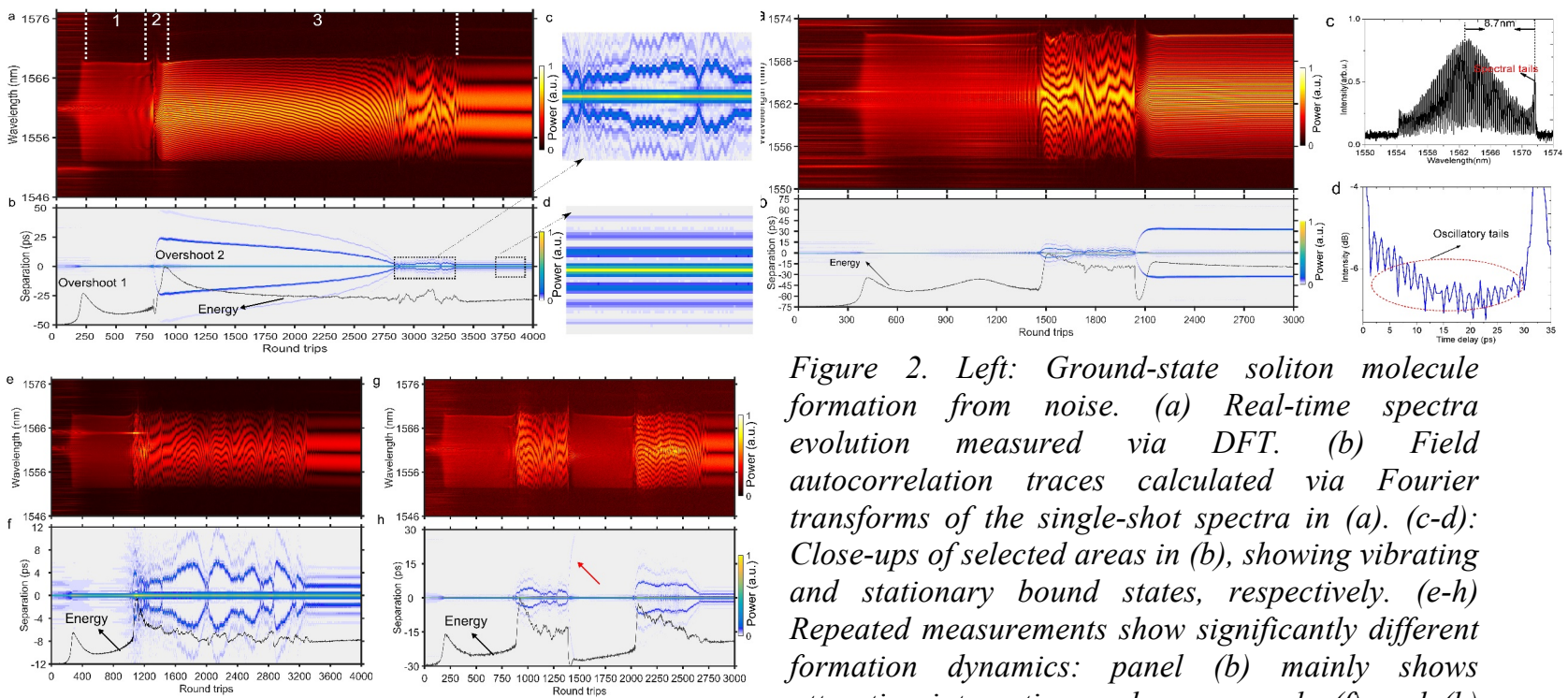

Figure 2. Left: Ground-state soliton molecule formation from noise. (a) Real-time spectra evolution measured via DFT. (b) Field autocorrelation traces calculated via Fourier transforms of the single-shot spectra in $(a)$. (c-d): Close-ups of selected areas in (b), showing vibrating and stationary bound states, respectively. $(e-h)$ Repeated measurements show significantly different formation dynamics: panel (b) mainly shows attractive interactions, whereas panels $(f)$ and $(h)$ feature vibration and annihilation, respectively. Right: Excited-state soliton molecule formation. (a) Measured DFT data. (b) Corresponding field autocorrelation traces, showing that repulsive interactions dominate the final stage of the build-up. (c-d): Cross-sections of (a) and (b) at the last cavity round trip, showing oscillatory tails in both the frequency and time domains.

\subsection{Soliton molecules in normal-dispersion cavities}

Soliton molecules have been intensively studied in anomalous-dispersion fibre lasers. In [9], we report on the first real-time observation of dynamical soliton molecules in the normal-dispersion regime of a laser cavity by using high-resolution DFT measurements and field autocorrelation analysis assisted by spatio-temporal intensity measurements. We observe an aperiodic evolution of the intra-molecular temporal separation over cavity round trips. The elementary solitons within the molecule exchange energy during propagation, and the stronger soliton splits when the energy exchange occurs. Moreover, rogue waves are present in the molecule spectrum. Unlike anomalous-dispersion cavities, no soliton molecules with fixed intra-pulse separation and phase are found at 
normal dispersion. Such distinctive features of soliton molecules in cavities with normal dispersion are attributed to the high energies of dissipative solitons in such cavities, as well as to pronounced dissipative effects such as gain filtering.

\subsection{Collision-induced soliton explosions}

Soliton explosions are among the most striking nonlinear dissipative phenomena that can manifest in modelocked lasers. In this regime, a dissipative soliton circulating in the laser cavity experiences an abrupt structural collapse, but within a few round trips returns to its original quasi-stable state. Such events have been recently observed in a mode-locked fibre laser [14]. While for long-cavity lasers, Raman scattering seems to relate to soliton explosions, for short-cavity lasers the origins of soliton explosions have remained elusive. Benefiting from synchronous real-time spatio-temporal intensity and DFT-based spectrum measurements, we demonstrate that soliton collisions can induce soliton explosions [10]. Up to seven nonlinear regimes are observed successively in the laser by solely increasing the pump strength, including single-pulse mode locking, standard soliton explosions, noise-like mode locking, stable double pulsing, collision-induced soliton explosions, soliton molecules, and double-pulse noise-like mode locking. The long-range attractive interactions of solitons in our normal-dispersion laser are deemed to arise from gain depletion and recovery.

\section{DYNAMICS OF BREATHING DISSIPATIVE SOLITONS}

\subsection{Generation of breathers and breather molecular complexes}

Dissipative breathers have been first demonstrated in passive Kerr cavities, and subsequently observed in optical micro-resonators [15]. In [11], we report on the direct experimental observation of breathing solitons in a modelocked fibre laser. Breathers feature synchronous periodic variations of their temporal intensity and spectrum over cavity round trips. Such fast evolutionary behaviour is captured in real time by spatio-temporal intensity and DFT-based measurements. In the normal-dispersion regime of the laser cavity breathers are excited in the laser under the pump threshold for stationary mode locking. Our work first establishes a general, deterministic route to induce soliton breathing in normal-dispersion fibre cavities in that the breather generation regime is accessible by solely changing the pump strength. Besides, for the first time in experiments with mode-locked fibre lasers, breathing soliton pair molecules are also generated in the cavity.

While soliton pairs constitute the central soliton molecule case, soliton molecules can exist in various isomers, and a large population of optical solitons can self-assembly into macromolecules and soliton crystals [16]. In [12], by tuning the cavity loss through small rotations of the polarisation controllers at fixed pump strength in an anomalous-dispersion mode-locked laser, we demonstrate various types of breather complexes. These include multi-breather molecules (Fig. 3, left panel), and molecular complexes formed by the binding of two basic molecules, each made up of a pair of breathing solitons, or a breather-pair molecule and a single breather. The inter-molecular temporal separation of such complexes is more than an order of magnitude larger than that of their stationary soliton counterparts, and is a signature of long-range interactions. We also explore the nonequilibrium dynamics of breather molecular complexes, including collisions of breathers and annihilation of an elementary breather within a complex.

\subsection{Breather explosions}

Pushing the similarity in behaviour between breathing and stationary solitons further, we demonstrate the explosive dynamics of breathers in a mode-locked fibre laser [13] (Figure 3, right panel). Different laser regimes, including breather explosions, stable breathers, and continuous-wave mode locking, are accessible by varying the pump power in the normal-dispersion laser cavity. In contrast to soliton explosions that are observed above the pump level for generating stable solitons, breathing soliton explosions occur under the pump threshold for stable breather mode locking. Nonlinearity-mediated giant $Q$-switching is the mechanism that triggers such explosions. Moreover, rogue waves are observed during the explosion events.

\section{CONCLUSIONS}

Ultrafast mode-locked lasers that exploit nonlinearity in the pulse formation process display a rich landscape of dissipative soliton dynamics, resulting from the interplay of the nonlinearity with dispersion and dissipation. We have reported on the experimental observation and real-time characterisation of various recent examples of such dynamics. Our observations are corroborated by numerical simulation of the laser model (the details will be presented at the conference). These findings contribute to the fundamental understanding of complex nonlinear wave dynamics relevant to a large variety of physical systems, and they carry also importance from an application perspective.

\section{ACKNOWLEDGEMENTS}

We acknowledge the support of the National Natural Science Fund of China $(11621404,11561121003,11727812$, 61775059, and 11704123), National Key Research and Development Program (2018YFB0407100), Key Project of Shanghai Education Commission (2017-01-07-00-05-E00021), and Science and Technology Innovation Program of Basic Science Foundation of Shanghai (18JC1412000). 

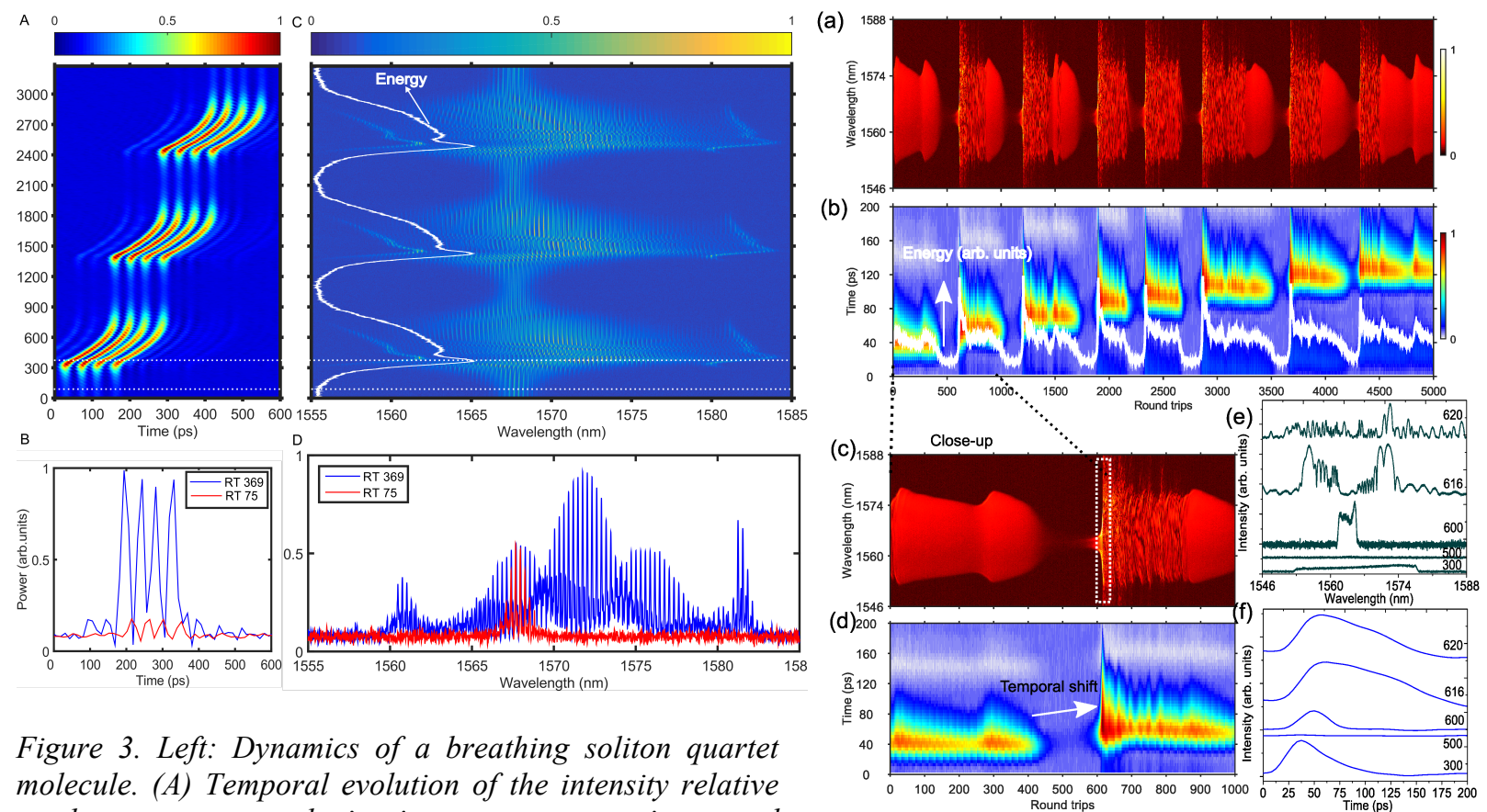

Figure 3. Left: Dynamics of a breathing soliton quartet molecule. (A) Temporal evolution of the intensity relative to the average round-trip time over consecutive round

trips. (B) Temporal intensity profiles at representative round-trip numbers of maximal and minimal energies within a period of oscillation. (C) DFT recording of single-shot spectra. The evolution of the energy is also shown (solid white curve). (D) Single-shot spectra at representative round-trip numbers of maximal and minimal spectrum extents within a period. Right: Dynamics of breathing soliton explosions. (a) Spectral evolution over consecutive round trips. (b) Synchronous temporal intensity evolution; the white curve shows the energy evolution. (c-d) Close-ups of selected areas in (a) and (b). (e-f) Representative cross-sections of (c) and (d) taken at the round trips 300, 500, 600, 616 and 620 .

\section{REFERENCES}

[1] P. Grelu, N. Akhmediev: Dissipative solitons for mode-locked lasers. Nat. Photon., vol. 6, pp. 84-92, 2012.

[2] G. Herink, et al:: Real-time spectral interferometry probes the internal dynamics of femtosecond soliton molecules. Science, vol. 356, pp. 50-54, 2017.

[3] K. Krupa, et al.: Real-time observation of internal motion within ultrafast dissipative optical soliton molecules. Phys. Rev. Lett., vol. 118, 243901, 2017.

[4] P. Ryczkowski, et al.: Real-time full-field characterization of transient dissipative soliton dynamics in a mode-locked laser. Nat. Photon., vol. 12, pp. 221-227, 2018.

[5] X. Liu, X. Yao, Y. Cui: Real-time observation of the buildup of soliton molecules. Phys. Rev. Lett., vol. 121, 023905,2018

[6] C. Bao, et al.: Observation of Fermi-Pasta-Ulam recurrence induced by breather solitons in an optical microresonator. Phys. Rev. Lett., vol. 117, 163901, 2016.

[7] J. Peng, et al.: Real-time observation of dissipative soliton formation in nonlinear polarization rotation mode-locked fibre lasers. Commun. Phys., vol. 1, 20, 2018.

[8] J. Peng, H. Zeng: Build-up of dissipative optical soliton molecules via diverse soliton interactions. Laser Photon. Rev., vol. 12, 1800009, 2018.

[9] J. Peng, H. Zeng: Dynamics of soliton molecules in a normal-dispersion fiber laser. Opt. Lett., vol. 44, pp. 2899-2902, 2019.

[10] J. Peng, H. Zeng: Soliton collision induced explosions in a mode-locked fibre laser. Commun. Phys., vol. 2 , 34, 2019.

[11]J. Peng, et al.: Breathing dissipative solitons in mode-locked fiber lasers. Sci. Adv., vol. 5, eaax1110, 2019.

[12] J. Peng, et al.: Breather molecular complexes in a passively mode-locked fibre laser. Submitted, 2020.

[13] J. Peng, H. Zeng: Experimental observations of breathing dissipative soliton explosions. Phys. Rev. Appl., vol. 12, 034052, 2019.

[14] A.F.J. Runge, N.G.R. Broderick, M. Erkintalo: Observation of soliton explosions in a passively modelocked fiber laser. Optica, vol. 2, pp. 36-39, 2015.

[15] M. Yu, et al.: Breather soliton dynamics in microresonators. Nat. Commun., vol. 8, 14569, 2017.

[16] A. Haboucha, et al.: Analysis of soliton pattern formation in passively mode-locked fiber lasers. Phys. Rev. $A$, vol. 78, 043806, 2008. 